Revue internationale de l'économie sociale

Recma

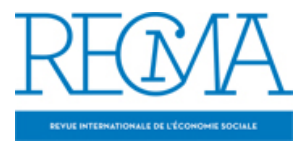

\title{
Les coopératives agricoles
}

Un atout pour la pérennité de l'agriculture dans la

mondialisation

\section{Agricultural cooperatives and the future of farming in a global economy}

\section{René Mauget}

Numéro 307, février 2008

Les coopératives d'entreprises

Entreprise Cooperatives

URI : https://id.erudit.org/iderudit/1021194ar

DOI : https://doi.org/10.7202/1021194ar

Aller au sommaire du numéro

Éditeur(s)

Association Recma

ISSN

1626-1682 (imprimé)

2261-2599 (numérique)

Découvrir la revue

Citer cet article

Mauget, R. (2008). Les coopératives agricoles : un atout pour la pérennité de l'agriculture dans la mondialisation. Revue internationale de l'économie sociale, (307), 46-57. https://doi.org/10.7202/1021194ar
Résumé de l'article

La coopération agricole a connu de profondes mutations dans les quarante dernières années. L'article rappelle les fondements historiques et juridiques des coopératives agricoles, ainsi que la place importante qu'elles occupent aujourd'hui dans la vie économique. Quelles sont les politiques financières déployées ? Quelles stratégies de développement - concentration, intégration verticale, diversification, internationalisation - les coopératives ont-elles mises en oeuvre ? Des groupes coopératifs complexes ont vu le jour, s'appuyant sur des filiales de statuts non coopératifs et des alliances avec des entreprises capitalistes. L'auteur analyse les conséquences de ces mutations, tant pour les adhérents qu'en termes d'efficacité de la coopérative. 


\title{
LES COOPÉRATIVES AGRICOLES
}

\section{Un atout pour la pérennité de l'agriculture dans la mondialisation}

\author{
par René Mauget $\left.{ }^{*}\right)$
}

La coopération agricole a connu de profondes mutations dans les quarante dernières années. L'article rappelle les fondements historiques et juridiques des coopératives agricoles, ainsi que la place importante quelles occupent aujourd'hui dans la vie économique. Quelles sont les politiques financières déployées? Quelles stratégies de développement - concentration, intégration verticale, diversification, internationalisation - les coopératives ont-elles mises en auvre? Des groupes coopératifs complexes ont vu le jour, s'appuyant sur des filiales de statuts non coopératifs et des alliances avec des entreprises capitalistes. L'auteur analyse les conséquences de ces mutations, tant pour les adhérents qu'en termes d'efficacité de la coopérative.

\section{Les fondements historiques des coopératives agricoles et les facteurs de développement}

La coopération agricole, en tant qu'expression de la solidarité paysanne, a des origines fort lointaines, mais l'organisation et la concentration au sein du mouvement sont apparues au début du siècle. L'indépendance et l'individualisme ont toujours été dans le monde paysan un sentiment très vif, mais il semble que les contraintes économiques et sociales aient amené les agriculteurs à se grouper et à s'entraider pour résoudre les difficultés résultant de leurs conditions de vie souvent difficiles.

Les fruitières des Alpes et du Jura, créées au XII siècle, peuvent être considérées comme les premières sociétés s'apparentant aux coopératives agricoles actuelles. Leur but était, en raison de la difficulté des communications dans les hautes vallées, la mise en commun du lait (collecte) et la fabrication d'un fromage de conservation facile (transformation et commercialisation), le gruyère.

De 1880 à 1890 furent créés de nombreux syndicats agricoles auxquels la loi du 21 mars 1884 donna une base légale, ce qui en favorisa l'éclosion. Ils se mirent très vite à effectuer des opérations d'achat en commun (notamment d'engrais, exerçant ainsi le rôle actuellement dévolu aux coopératives agricoles d'approvisionnement).

Notons qu'au XIX siècle le mouvement coopératif en agriculture s'est développé indépendamment du mouvement ouvrier coopératif ; ce n'est que plus tard que celui-ci eut des répercussions parmi les sociétés agricoles. D'autres exemples montrent que les agriculteurs sont venus à la coopération 
dans des périodes difficiles. Les ravages provoqués par le phylloxéra entraînèrent le remplacement des vignes par des prairies dans la région des Charentes, qui vit se créer les premières beurreries coopératives (les agriculteurs se groupèrent afin d'assurer des débouchés à la nouvelle production laitière). C'est en raison de la mévente des vins qu'apparurent les premières coopératives vinicoles dans l'Hérault. On peut toutefois noter qu’à cette période les relations avec les coopératives de consommateurs se développent, notamment dans le sud de la France. La crise de surproduction des années 30 conduisit à la création de nombreuses coopératives de stockage de céréales.

Le mouvement coopératif s'est ainsi implanté dans l'ensemble de la France et s'est étendu aux principaux secteurs de l'activité agricole.

\section{Evolution quantitative des coopératives depuis quarante ans}

\section{Chiffres clés}

Le tableau 1 présente l'évolution des chiffres clés des coopératives agricoles depuis 1965 .

\section{Tableau 1}

Evolution des coopératives agricoles depuis 1965

\begin{tabular}{l|r|r|r|r|r} 
Année & $\mathbf{1 9 6 5}$ & $\mathbf{1 9 7 2}$ & $\mathbf{1 9 7 8}$ & $\mathbf{2 0 0 0}$ & $\mathbf{2 0 0 6}$ \\
\hline $\begin{array}{l}\text { Nombre } \\
\text { d'organismes }\end{array}$ & 5717 & 4700 & 4141 & 3700 & $3200^{(1)}$ \\
\hline $\begin{array}{l}\text { CA (milliards } \\
\text { de francs ou d'euros) }\end{array}$ & $20,6 \mathrm{MdF}$ & $50 \mathrm{MdF}$ & $110 \mathrm{MdF}$ & $\begin{array}{r}65 \mathrm{Md} \\
(425 \mathrm{MdF})\end{array}$ & $\begin{array}{r}78 \mathrm{Md} € \\
(511 \mathrm{MdF})^{(2)}\end{array}$ \\
\hline Salariés & 80211 & 95700 & 107000 & 150000 & $150000^{(2)}$ \\
\hline & $\begin{array}{l}\text { Source: } \\
\text { SCEES }\end{array}$ & $\begin{array}{l}\text { Source: } \\
\text { SCEES }\end{array}$ & $\begin{array}{r}\text { Source: } \\
\text { SCEES }\end{array}$ & $\begin{array}{r}\text { Source: } \\
\text { Coop } \\
\text { de France }\end{array}$ & $\begin{array}{r}\text { Source: } \\
\text { Coop } \\
\text { de France }\end{array}$
\end{tabular}

(1) Plus 12500 Cuma.

(2) Y compris environ 1600 filiales.

Part globale des coopératives dans les filières

La coopération agricole est majoritaire dans la collecte des matières premières agricoles. Présente dans les première et deuxième transformations, ses parts de marchés sont moindres dans les produits élaborés (voir le tableau 2, en page suivante). 


\section{Tableau 2}

\section{Parts de marché par filières}

Production

Parts de marché

Pôle animal

Nutrition animale

$60 \%$

Aviculture, foie gras

Bétail viande

- porc (production)

- bovins (production)

Lait

- collecte

- transformation environ $45 \%$

Insémination animale

- bovins

- caprins

Pôle végétal

Betteraves, sucre

$62 \%$

Grains, collecte $75 \%$

Approvisionnement $70 \%$

Forêt $23 \%$

Lin (teillage)

$56 \%$

\section{Cultures spécialisées}

Vin

- AOC

$40 \%$

- vin de pays

$70 \%$

- champagne

$30 \%$

Fruits et légumes

- fruits frais

- légumes frais

- quatrième gamme

Tabac $100 \%$

Miel $20 \%$

\section{Les principaux groupes coopératifs français}

Les vingt premiers groupes coopératifs présentés dans le tableau 3 (voir en page suivante) représentent près de $35 \%$ du chiffre d'affaires de l'ensemble des coopératives agricoles françaises; ils constituent les principaux pôles de restructuration régionaux ou nationaux. 


\begin{tabular}{|c|c|c|c|c|c|}
\hline & Entreprises & Coordonnées & $\begin{array}{l}\text { Activités } \\
\text { principales }\end{array}$ & $\begin{array}{l}\text { Marques } \\
\text { principales }\end{array}$ & $\begin{array}{l}\text { CA consolidé } \\
2006(\mathrm{M} €)\end{array}$ \\
\hline 1 & Terrena & 44150 Ancenis & Polyvalente & $\begin{array}{l}\text { Gastronome, } \\
\text { Paysan breton, } \\
\text { Soviba, Val d'Ancenis }\end{array}$ & 3100 \\
\hline 2 & In Vivo & 75782 Paris & $\begin{array}{l}\text { Céréales } \\
\text { approvisionnement }\end{array}$ & $\begin{array}{l}\text { Gamm vert, } \\
\text { Semences de France }\end{array}$ & 2800 \\
\hline 3 & Tereos & 02390 Origny & Sucre & $\begin{array}{l}\text { Beghin-Say, L'Antillaise, } \\
\text { La Perruche, Origny }\end{array}$ & 2277 \\
\hline 4 & Sodiaal & 75014 Paris & Lait & $\begin{array}{l}\text { Yoplait, Candia, } \\
\text { Riches Monts, Nactalia }\end{array}$ & 1955 \\
\hline 5 & Socopa & 75755 Paris & Viande & $\begin{array}{l}\text { Hit Burger, Val tendre, } \\
\text { Valtero }\end{array}$ & 1940 \\
\hline 6 & Agrial & 14050 Caen & Polyvalente & $\begin{array}{l}\text { Florette, Manon, Priméale, } \\
\text { Prim'Co }\end{array}$ & 1520 \\
\hline 7 & $\begin{array}{l}\text { Coopagri } \\
\text { Bretagne }\end{array}$ & 29206 Landerneau & Polyvalente & $\begin{array}{l}\text { Paysan breton, } \\
\text { Prince de Bretagne, Régilait, } \\
\text { Ronsard }\end{array}$ & 1453 \\
\hline 8 & Unicopa & 29210 Morlaix & Polyvalente & $\begin{array}{l}\text { Cuisine et Vrai, } \\
\text { Brocéliande, Rippoz }\end{array}$ & 1400 \\
\hline 9 & Cecab & 56004 Vannes & Polyvalente & D’Aucy & 1313 \\
\hline 10 & $\begin{array}{l}\text { Champagne } \\
\text { Céréales }\end{array}$ & 51059 Reims & $\begin{array}{l}\text { Céréales } \\
\text { approvisionnement }\end{array}$ & Banette, Francine & 1103 \\
\hline 11 & Cristal Union & $\begin{array}{l}45490 \text { Corbeilles- } \\
\text { en-Gâtinais }\end{array}$ & Sucre & Daddy & 1100 \\
\hline 12 & Limagrain & 63720 Ennezat & Semences, bio-santé & $\begin{array}{l}\text { Limagrain, Vilmorin, } \\
\text { Clause, Pain Jacquet }\end{array}$ & 1093 \\
\hline 13 & $\begin{array}{l}\text { Cooperl } \\
\text { Hunaudaye }\end{array}$ & 22403 Lamballe & Viande & Calidel & 1008 \\
\hline 14 & Epis-Centre & 18028 Bourges & $\begin{array}{l}\text { Céréales } \\
\text { approvisionnement, } \\
\text { meunerie, malt }\end{array}$ & & 964 \\
\hline 15 & Groupe Even & 29260 Ploudaniel & $\begin{array}{l}\text { Lait, } \\
\text { approvisionnement, } \\
\text { nutrition animale }\end{array}$ & $\begin{array}{l}\text { Even, Paysan breton, } \\
\text { Mamie Nova, Kerguelen }\end{array}$ & 893 \\
\hline 16 & Euralis & 64230 Lescar & Polyvalente & $\begin{array}{l}\text { Semences Rustica, } \\
\text { Montfort-Grimaud, Rougié-Bizac }\end{array}$ & $\mathrm{zac}$ \\
\hline 17 & Maïsadour & $\begin{array}{l}40001 \text { Mont- } \\
\text { de-Marsan cedex }\end{array}$ & Polyvalente & Delpeyrat, Sarrade & 617 \\
\hline 18 & $\begin{array}{l}\text { Alliance } \\
\text { agro-alimentaire }\end{array}$ & 31016 Toulouse & Lait & Cantorel, Capitoul, Pilpa & 616 \\
\hline 19 & Unéal & $\begin{array}{l}62054 \text { Saint-Laurent- } \\
\text { Blangy cedex }\end{array}$ & Polyvalente & & 588 \\
\hline 20 & Agralys & 28201 Châteaudun & $\begin{array}{l}\text { Céréales } \\
\text { approvisionnement }\end{array}$ & & 540 \\
\hline \multicolumn{6}{|c|}{ Source: rapports annuels-presse. } \\
\hline
\end{tabular}




\section{Statut et gouvernance des coopératives}

\section{Loi de 1972}

La loi du 27 juin 1972 définit la société coopérative agricole comme un groupement professionnel qui a les caractéristiques suivantes:

- société à capital variable;

- société de personnes;

- société juridiquement spécifique, différente des sociétés civile et commerciale. Le statut défini par la loi de 1972 est assorti d'options.

- Option économique: dérogation à l'exclusivisme (la coopérative peut réaliser des opérations avec des non-coopérateurs).

- Options financières: associés non coopérateurs; réévaluations des bilans; revalorisation du capital social.

- Options gouvernance: pondération des voix; gestion pour directoire et conseil de surveillance.

- Utilisation en commun par les agriculteurs de tous moyens propres à faciliter ou à développer l'activité économique de sociétaires et à accroître les résultats de leur activité.

\section{Evolution des spécificités juridiques}

Les lois de 1972, 1991 et 1992 ont aménagé partiellement les contraintes économiques et financières. Par ailleurs, la politique de filialisation a facilité le partenariat financier (holding), le partenariat industriel et commercial avec les firmes capitalistes: remontée des dividendes à l'agriculteur (loi 1991). La loi de 1999 sur la consolidation des comptes a conduit à davantage de transparence financière. La loi du 6 janvier 2006, à la suite du rapport Guillaume, a pour ambition de renforcer l'information et le pouvoir des agriculteurs et d'inciter à une plus grande redistribution des surplus aux adhérents au détriment de la mise en réserve des excédents.

\section{Régime fiscal}

Les opérations réalisées avec les sociétaires sont exonérées de l'impôt sur les sociétés. Les activités avec les tiers et les filiales sont fiscalisées.

La base retenue pour la taxe professionnelle est réduite de moitié. Certaines coopératives en sont exemptées: les coopératives employant trois salariés au plus; les coopératives de vinification; les coopératives de fruits et légumes, d'insémination animale et les Cuma.

\section{Stratégies et enjeux des coopératives}

\section{Les stratégies de développement La stratégie de concentration}

La concentration a été importante sur les quarante dernières années. La chasse aux parts de marché et aux économies d'échelle reste un objectif 
pour de nombreux groupes. Cependant, les études montrent que la concentration à elle seule ne suffit pas à assurer la rentabilité (encore faudrait-il définir ce concept dans le cas coopératif, qui n’a pas les mêmes buts qu'une société de capitaux). Il convient donc de bien concentrer les fonctions qui doivent l'être (collecte, outil industriel, commercialisation, recherche, finance, etc.) pour réduire les coûts ou améliorer l'efficacité.

Si la concentration est souvent nécessaire par rapport aux pressions de l'environnement, pour les produits de masse, il existe encore de nombreuses opportunités pour des entreprises plus petites qui vont travailler sur des segments de marché différents.

Cette concentration a été essentiellement effectuée de deux façons: par regroupement de coopératives (le plus fréquent) ou par rachat d'entreprises privées (plus coûteux).

Cependant, les grandes manœuvres dans la distribution, la globalisation des marchés et l'internationalisation des concurrents vont conduire les coopératives à accélérer le processus de concentration.

\section{La stratégie d'intégration verticale}

Cette stratégie s'est traduite dans les campagnes par deux slogans: "Récupérons la valeur ajoutée » ou, dans les années 70, «Suivons le bœuf ». L'intégration verticale a été largement développée dans les secteurs de production animale (lait, viande); peu de coopératives se sont limitées à la collecte. En revanche, en céréales, l'intégration verticale n’a pas été très poussée, mis à part quelques groupes (Champagne Céréales notamment).

Depuis quelques années, on assiste à des désengagements forcés avec un retour au métier de base (ULN, Arcadie, Transagra, France Volailles). Dans ces retraits, les coopératives ont parfois conservé une participation minoritaire dans la société repreneuse.

Il convient de signaler que, dans la plupart de ces cas, si le contrôle a été perdu, les droits à produire avec un contrat incluant des clauses dissuasives peuvent résister, mais les risques de délocalisation des productions sont plus grands. La notion de taille critique doit également être considérée pour chaque maillon de la chaine d'intégration.

En conclusion, les stratégies d'intégration verticale ne sont pas fermées aux coopératives, mais elles devront être étudiées attentivement dans cet environnement plus difficile et envisagées sous forme partenariale, notamment avec le privé.

Les coopératives pouvant autofinancer seules l'aval sont de plus en plus rares. De plus, il faut maîtriser les techniques et les marchés; c'est là que le partenariat industriel, commercial et financier peut apporter les facteurs de réussite, mais il ne constitue pas non plus une assurance tous risques (voir la faillite de Bourgoin).

\section{La diversification est-elle la panacée?}

Plusieurs études conduites ces dernières années auprès des coopératives évoquaient la diversification comme solution aux chocs PAC (1984 et 
1992). Depuis, on assiste à des recentrages sur le ou les métiers de base plutôt qu'à des diversifications. Les groupes polyvalents de l'ouest de la France ont su également se restructurer autour de trois ou quatre métiers et se concentrer par partenariat autour de ces métiers.

Il existe néanmoins quelques cas de diversification majeure: Pau-Euralis ou Maïsadour, qui ont effectué des alliances avec le privé (p. ex. Bonduelle) ou des rachats d'entreprise (p. ex. foie gras); la Casam (aujourd'hui Agrial), qui s'est diversifiée sur les marchés des légumes et de la viande à partir d'une coopérative d'approvisionnement.

La diversification demande des moyens financiers, des compétences techniques, un marché. Là encore, pour acquérir ces atouts, le partenariat est souvent une solution plus rapide et moins risquée.

\section{Les stratégies d'internationalisation}

Nous avons vu que les marchés et les concurrents privés s'internationalisaient. Les coopératives exportent, mais sont peu internationalisées (implantation à l'étranger). En effet, l'internationalisation n'est pas toujours bien perçue par l'adhérent, qui craint parfois à juste titre que celle-ci soit négative pour lui: limitation des droits à produire, réimportation de produits moins chers... Cependant, sur le moyen terme, les concurrents qui optimisent leur rentabilité par leur couverture internationale pourront posséder un avantage compétitif face à des coopératives, surtout lorsqu'il existe des barrières à l'exportation.

En France, une trentaine de groupes coopératifs ont conduit des stratégies d'internationalisation (Sodiaal, Limagrain, Champagne Céréales, Cecab, Agrial, Tereos, etc).

L'enjeu de l'internationalisation implique des choix qui sont très souvent difficiles à faire par les responsables de coopératives et les résultats attendus ne sont pas toujours au rendez-vous et parfois difficiles à expliquer aux coopérateurs.

\section{Les politiques financières déployées par les coopératives}

Nous avons évoqué précédemment l'importance de ce facteur dans l'évolution des coopératives françaises. En effet, la faible capacité d'autofinancement (CAF) par rapport aux industries agro-alimentaires (IAA) privées est souvent la conséquence d'une valeur ajoutée plus faible. A titre d'exemple, au début des années 2000, le taux moyen de valeur ajoutée des firmes privées présentes dans les IAA était nettement supérieur à celui des coopératives dans la même branche, respectivement $20 \%$ et $13 \%$. Cette faible CAF engendre une faible capacité de croissance autofinancée, donc un recours plus important au financement extérieur. Par ailleurs, nous voyons qu'en France, notamment, les agriculteurs financent à un faible niveau leurs outils coopératifs; il en découle donc une nécessité de financer davantage par l'emprunt. Cette solution, parfaitement acceptable lorsque le taux d'intérêt réel était négatif ou très faible (effet de levier), devient dangereuse avec des taux d'intérêt réels élevés (effet de massue). Plusieurs difficultés 
(1) Ce paragraphe constitue un résumé de la contribution de René Mauget et de Michel Forestier au XVe colloque Addes (mars 2000). Voir aussi R. Guillouzo, P. Perrot, P. Ruffio, "Stratégies d'alliances et configurations des groupes coopératifs agricoles ", Recma, $\mathrm{n}^{\circ} 285$ 2002 de coopératives entre 1985 et 1995 peuvent s'expliquer en partie de cette façon. Actuellement, les taux réels sont revenus à un niveau acceptable et les banques redisent à loisir qu'il n’y a pas de difficulté à financer de bons projets.

Le texte sur les titres participatifs et la loi à dominante financière du 3 janvier 1991 n'ont pas apporté de solutions satisfaisantes à l'insuffisance de capitaux propres, car ces capitaux sont chers pour l'entreprise et pas très attrayants pour l'apporteur. Une dizaine de coopératives auraient utilisé les titres participatifs dont deux en Bourse (Cana, ULN). En revanche, les autres produits (CCI, obligations) ne sont pas ou peu utilisés. En 2007, Tereos a levé 500 millions d'euros d'obligations pour de la croissance externe. Les systèmes de portage mis en place par les banques, soit au niveau de la société mère, soit au niveau des filiales, sont également utilisés par les groupes coopératifs.

L'entrée en Bourse des filiales de coopérative est possible, mais à ce jour l'image et la rentabilité de ces filiales n'ont pas permis de lancer ce type d'opération, si ce n'est le cas de la filiale du groupe Limagrain (Vilmorin) et celui de Tereos avec sa filiale au Brésil. Seules les coopératives irlandaises semblent à ce jour avoir bien réussi leur pénétration sur le marché boursier en Europe. Cependant, cette entrée en Bourse a eu pour corollaire la perte de pouvoir des agriculteurs dans leur coopérative, qui a désormais d'autres objectifs économiques et financiers.

Face à ces problèmes de financement pour les groupes agro-alimentaires, les stratégies partenariales sont en général plus économes en capitaux, ce qui explique leur développement, même si ces dernières ont d'autres raisons d'être. Dans les prochaines années, nous verrons sans doute se développer un financement des agriculteurs dans les filiales des coopératives. Quelques expériences germent aujourd'hui (p. ex., groupe Epis-Centre et Agrial).

A l'issue de cette approche, il convient cependant de noter que de nombreuses coopératives ont des situations financières saines et n'ont pas de gros besoins de capitaux. Notre analyse s'applique surtout aux groupes ayant des ambitions de croissance dans l'agro-alimentaire et l'agro-industrie.

\section{Les mutations structurelles: causes et conséquences ${ }^{(1)}$}

Les coopératives agricoles existent de moins en moins à l'état pur. Elles constituent, notamment lorsqu'elles transforment leurs produits, des groupes qui, autour d'une coopérative, articulent des filiales de statuts non coopératifs. Les filiales sont en outre souvent détenues en commun avec d'autres coopératives, voire des sociétés capitalistes industrielles ou bancaires. Se trouvent ainsi combinées dans les faits des logiques différentes, coopérative et capitaliste. Pour l'une, le facteur risqué est le produit, pour l'autre, il s'agit du capital.

\section{Pourquoi ces évolutions?}

Avant d'analyser les conséquences, il est intéressant de revenir sur le pourquoi de ces évolutions. Nous avons identifié au moins cinq types de raisons. 
(2) En droit, deux fois le capital social.

(3) Dans la pratique, la loi de 1972, en France, permet de revaloriser le capital social avec les réserves disponibles, dans la limite de l'évolution de la rente viagère.
- Des raisons stratégiques consécutives à une globalisation des marchés. Cette globalisation a presque toujours eu comme conséquence une concentration, notamment commerciale, mais aussi une nécessité de réduire les coûts et (ou) de différencier les produits.

- Des raisons juridiques: contraintes statutaires limitant la capacité (règle de l'exclusivisme et de territorialité, notamment); contraintes pour faire des alliances entre le secteur privé et la société mère coopérative.

- Des raisons financières: les difficultés rencontrées pour mobiliser les capitaux propres avec la société mère entraînent le développement des holdings.

- Des raisons fiscales parfois: la structure holding permet de bénéficier de l'intégration fiscale avec les résultats des filiales.

- Des raisons de management, de prise de décision et de gestion: simplification, rapidité, meilleure vision des rentabilités par métier, facilitation du recentrage, etc.

Il convient cependant de noter que la filialisation n'est pas la réponse unique à ces problèmes ni une panacée.

En résumé, on peut dire que le but de cette évolution était et est toujours d'assurer la survie et (ou) d'augmenter l'efficacité du groupe coopératif face à l'accroissement de la dynamique concurrentielle.

Aujourd'hui, après une vingtaine d'années de filialisation, il est intéressant de s'interroger sur les conséquences de ces mouvements.

\section{Conséquences de ces mutations}

Les conséquences sont envisagées en matière de rémunération des agriculteurs et de critères d'efficacité.

\section{Dans la coopérative agricole classique}

Dans la coopérative agricole classique, le facteur risqué est le produit, alors que dans une entreprise capitaliste, il s'agit du capital. Le capital social dans la coopérative est plus proche d'un capital-obligations ou d'un prêt des membres que d'un capital-actions. Il subit néanmoins le risque de faillite ${ }^{(2)}$. Les implications stratégiques et financières de cette forme d'organisation sont donc déterminantes.

Le capital bénéficie d'une rémunération limitée pouvant aller de zéro jusqu'au taux moyen des obligations (en France). Les sociétaires n'ont pas de droit sur les réserves ${ }^{(3)}$. En revanche, les surplus dégagés par l'exploitation peuvent être distribués aux adhérents sous forme de ristournes au prorata des transactions économiques avec la coopérative. C'est le principe « acapitaliste ".

Ainsi, la valeur de la coopérative pour l'adhérent se manifeste d'abord par l'équation: (acompte + complément de prix + ristournes) x quantités produites. A cela on peut ajouter la rémunération du capital social.

L'approche patrimoniale de la coopérative pour l'adhérent, par le bilan, est également plus complexe à appréhender que dans l'entreprise capitaliste. En effet, l'adhérent n'a pas de droit sur les réserves, il n'a pas droit aux plus-values. La valeur de son capital reste à la valeur nominale comme 
dans le cas d'un prêt ou d'un compte associé; il n'a pas droit non plus à des dividendes.

\section{Cas du groupe complexe}

Les implications en matière de management stratégique et financier sont importantes pour la coopérative et pour l'agriculteur. En effet, dans un groupe complexe, surtout lorsqu'il y a des partenaires non coopératifs, qu'ils soient financiers (banque) ou industriels, nous avons un mariage entre deux entités qui souhaitent optimiser un facteur risqué différent: les agriculteurs, le produit; le partenaire capitaliste, le capital.

Pour résumer, la rémunération du produit dans cette sphère évolue de la façon suivante.

- Coopérative classique: (acompte + complément de prix + ristournes).

- Sphère aval dans les groupes complexes (le résultat est externalisé) : (prix de marché + dividendes), avec possibilité, depuis la loi du 3 janvier 1991 en France, de faire une remontée des dividendes jusqu'aux sociétaires et plus-values possibles si l'agriculteur investit dans les filiales.

Il reste que la société mère peut encore effectuer des arbitrages au sein de la coopérative entre intérêt sur le capital social, dividendes et ristournes. Des conflits peuvent apparaitre entre actionnaires sur les finalités, les valeurs, les stratégies et la répartition des résultats.

\section{Les relations adhérents-coopératives}

L'ensemble de ces évolutions économiques peut troubler la relation entre la coopérative et l'adhérent. L'un des enjeux majeurs de la coopération est sans doute d'accroître la culture du "marché » des adhérents - comme l'ont fait les coopératives du nord de l'Europe - et de permettre ainsi aux agriculteurs de comprendre et de jouer un rôle actif dans les évolutions des groupes coopératifs.

Sans doute cela nécessite-t-il aussi de la part des coopératives une meilleure pédagogie et une meilleure communication.

\section{Les enjeux de développement durable}

Les coopératives agricoles sont, plus qu'une entreprise capitaliste, liées au territoire, donc a priori plus préoccupées par le développement durable, même si les pratiques historiques n'ont pas toujours été protectrices de l'environnement.

"Le développement durable est un développement qui répond aux besoins $d u$

(4) Définition du rapport Bruntland (1987), commission des Nations unies sur l'environnement et le développement. présent sans compromettre la possibilité pour les générations à venir de pouvoir répondre à leurs propres besoins ${ }^{(4)}$. " Le concept repose sur une gouvernance équilibrée par le triptyque: préservation de l'environnement; développement social (satisfaction des besoins); développement économique (création de richesses).

Les coopératives agricoles, par leurs finalités et leurs spécificités juridiques, sont porteuses de développement durable. Les finalités, axées sur la pérennité des bassins de production et le revenu des agriculteurs adhérents, 
contribuent aux axes du développement durable. Le statut juridique, qui fixe la territorialité, l'engagement réciproque et le principe de la dévolution de l'actif net, renforce la stabilité du capital sur le long terme.

De plus, l'attitude vis-à-vis de l'environnement durable devrait être plus respectueuse que celle d'une entreprise qui se délocalise en fonction des opportunités de profit.

Face à l'augmentation de la demande mondiale, le slogan « Produire plus et mieux " va devenir une nécessité et remplacer l'ancien slogan "Produire moins et mieux ». Peut-on atteindre ce double objectif sans rupture technologique et sans changement de politique agricole? Les coopératives seront au cœur de cet enjeu.

Coop de France a placé le développement durable parmi ses priorités. Une politique de sensibilisation est développée et deux outils sont déployés auprès des coopératives: le bilan sociétal, déjà en place, et un outil sur les indicateurs de développement durable, en test.

Les coopératives veulent utiliser ce concept de développement durable comme un atout et montrer la modernité de cette forme d'organisation face à une mondialisation dont les repères et les stabilisateurs manquent. Le développement durable peut être un levier d'action et de communication pour améliorer l'image du secteur agro-coopératif et pour rapprocher le consommateur et le citoyen de ce secteur.

\section{Conclusion générale}

(5) Un adhérent de coopérative n'est pas un actionnaire: sa part est remboursée à la valeur nominale; il n'a pas droit aux réserves (sauf revalorisation). Il est plutôt un " usufruitier " de sa coopérative qu'un actionnaire de plein exercice.
A l'aube du XXI' siècle, on observe des groupes coopératifs complexes (filialisation, alliance avec des entreprises capitalistes, cotation en Bourse, etc.). Ces ensembles ne sont plus tout à fait comparables aux coopératives de village de la première moitié du XXe siècle. En effet, avec la pression de la globalisation des marchés et, plus récemment, avec la baisse des protections (PAC-OMC), nous avons assisté à une concentration importante des concurrents, des fournisseurs et des clients des coopératives. Celles-ci ont réagi par des stratégies de concentration, d'intégration verticale, de diversification, ainsi que par l'entrée de nouveaux moyens financiers dans le cadre de groupes complexes (coopérative mère, holding, filiales de droit commun). Aujourd'hui, ces groupes coopératifs conduisent leurs stratégies dans des réseaux complexes d'alliances entre coopératives, mais aussi avec des partenaires capitalistes, qu'ils soient industriels, commerciaux ou financiers. Ces groupes reprennent également des entreprises capitalistes qui se désengagent de France.

Dans ce nouveau contexte, les finalités évoluent: à côté de la valeur de la coopérative pour l'usager, on voit apparaitre la valeur de la coopérative pour l'adhérent via son capital ${ }^{(5)}$.

Les valeurs changent, l'équité a remplacé largement l'égalité entre les sociétaires. Les principes des " pionniers de Rochdale " sont bousculés, voire non adaptés, pour certains d'entre eux, dans l'environnement 
concurrentiel actuel (acapitalisme et exclusivisme, notamment). En effet, l'ouverture du capital à l'extérieur et le travail avec des non-sociétaires sont parfois une nécessité. La remontée des dividendes de filiales au prorata du capital constitue également une entorse au principe acapitaliste.

Il convient donc de donner un sens à ces nouvelles formes d'organisation, nécessaires face à la globalisation des marchés, mais dont la logique reste cependant différente de celle des entreprises capitalistes, notamment en matière de gouvernance et de finalités. 